PROCEEDINGS OF THE

AMERICAN MATHEMATICAL SOCIETY

Volume 133, Number 4, Pages 1057-1061

S 0002-9939(04)07803-7

Article electronically published on November 3, 2004

\title{
EMBEDDING $B_{\infty}$ INTO MUCKENHOUPT CLASSES
}

\author{
THEMIS MITSIS
}

(Communicated by Andreas Seeger)

\begin{abstract}
What is the smallest $p$ for which a weight in the reverse Hölder class $B_{\infty}$ also belongs to the Muckenhoupt class $A_{p}$ ? We give an asymptotically sharp answer to this question.
\end{abstract}

\section{INTRODUCTION}

Let $w$ be a weight, that is, a positive locally integrable function on $\mathbb{R}^{n}$. We say that $w$ belongs to the $B_{\infty}$ class if there exists a constant $C>0$ such that for all cubes $Q$ (here and in the rest of the paper, by the term cube we will always mean a cube with sides parallel to the coordinate axes) we have

$$
w(x) \leq C \frac{1}{|Q|} \int_{Q} w, \text { for almost all } x \in Q,
$$

where $|\cdot|$ denotes Lebesgue measure. The smallest such $C$ is called the $B_{\infty}$ constant of $w$ and is denoted by $B_{\infty}(w)$. Clearly, $B_{\infty}(w) \geq 1$.

This class was first defined by Franchi 3 and then extensively studied by CruzUribe and Neugebauer 2. Earlier the $B_{\infty}$ condition appeared in the papers by Andersen and Young [1] and Muckenhoupt [5].

Every $B_{\infty}$ weight satisfies a reverse Hölder inequality (of arbitrary exponent) and consequently belongs to a Muckenhoupt class $A_{p}$, for a certain $p, 1<p<\infty$. Recall that $A_{p}$ consists of those weights $w$ for which there exists $C>0$ such that for all cubes $Q$,

$$
\left(\frac{1}{|Q|} \int_{Q} w\right)\left(\frac{1}{|Q|} \int_{Q} w^{-\frac{1}{p-1}}\right)^{p-1} \leq C
$$

It is therefore of interest to try to determine the smallest possible $p$ for which a $B_{\infty}$ weight belongs to $A_{p}$. "Smallest" should be understood in the asymptotic sense, that is, as the $B_{\infty}$ constant of $w$ approaches 1 .

The purpose of this paper is to prove the following asymptotically sharp result.

Theorem. There exist positive constants $C_{n}, \widetilde{C}_{n}$, with $\widetilde{C}_{n}>1$, such that for every $w \in B_{\infty}$ with $B_{\infty}(w) \leq \widetilde{C}_{n}$ we have

$$
w \in A_{p}, \text { for all } p>1+C_{n} \log B_{\infty}(w) .
$$

Received by the editors October 14, 2003.

2000 Mathematics Subject Classification. Primary $42 \mathrm{~B} 25$.

(C)2004 American Mathematical Society Reverts to public domain 28 years from publication 1057 
The conclusion of the Theorem conforms with our intuition. As $B_{\infty}(w)$ approaches $1, w$ tends to be a constant function, and therefore, one expects it to tend to belong to every $A_{p}$ class.

To see why the Theorem is asymptotically sharp as well, consider the power weight

$$
w_{\rho}\left(x_{1}, \ldots, x_{n}\right)=\left|x_{1}\right|^{\rho}, \rho>0 .
$$

A calculation shows that

$$
\log B_{\infty}\left(w_{\rho}\right) \sim \rho, \text { for } \rho \leq 1
$$

Now, in order for $w_{\rho}$ to belong to $A_{p}$ we need $\rho<p-1$, which means

$$
p>1+C \log B_{\infty}\left(w_{\rho}\right) .
$$

\section{Notation, TERMinology AND PRELIMINARIES}

Throughout the paper the capital letter $C$ and its variants $C_{1}, C_{2}, \ldots$ denote various positive constants not necessarily the same at each of their occurrences. $C_{n}$ denotes constants depending on the dimension $n$, again not necessarily the same each time they occur.

If $d x$ is Lebesgue measure and $w$ is a weight, then $d w$ is the measure defined by $d w=w d x$. In that case, we write $w(A)$ instead of $d w(A)$ for any measurable set $A$.

We say that a weight $w$ is doubling if there exists $C>0$ such that for every pair of concentric cubes $Q \subset \widetilde{Q}$ with $|\widetilde{Q}|=2^{n}|Q|$ we have

$$
w(\widetilde{Q}) \leq C w(Q) .
$$

The smallest such $C$ is called the doubling constant of $w$, denoted by $D_{w}$. Every $B_{\infty}$ weight is doubling.

Let $w$ be a weight and $f$ a $d w$-locally integrable function. $f$ is said to be in the $A_{1}^{d w}$ class if there exists $C>0$ such that for all cubes $Q$,

$$
\frac{1}{w(Q)} \int_{Q} f d w \leq C f(x), \text { for } d w \text {-almost all } x \in Q .
$$

As before, the smallest $C$ for which the above inequality holds is denoted by $A_{1}^{d w}(f)$.

Finally, we say that $f$ is in $B M O(d w)$ if

$$
\|f\|_{B M O(d w)}:=\sup _{Q} \frac{1}{w(Q)} \int_{Q}\left|f-f_{Q}\right| d w<\infty
$$

where

$$
f_{Q}=\frac{1}{w(Q)} \int_{Q} f d w
$$

is the average of $f$ on $Q$ with respect to $d w$. The symbol $d w$ in the notation introduced above will be suppressed if $w=1$.

A quantitative form of the relation between $B M O(d w)$ and $A_{1}^{d w}$ is given by the following result (see [4).

Theorem 2.1. Let $w$ be a doubling weight, and $f \in A_{1}^{d w}$. Then $\log f \in B M O(d w)$ and

$$
\|\log f\|_{B M O(d w)} \leq 2 \log A_{1}^{d w}(f) .
$$




\section{Proof of the Theorem}

Note to begin with, that since $w \in B_{\infty}$, it is clear that $w^{-1} \in A_{1}^{d w}$ with $B_{\infty}(w)=$ $A_{1}^{d w}\left(w^{-1}\right)$. Consequently, by Theorem 2.1 we have

$$
\|\log w\|_{B M O(d w)} \leq C \log A_{1}^{d w}\left(w^{-1}\right)=C \log B_{\infty}(w) .
$$

To estimate the unweighted $B M O$ norm of $\log w$, it is enough to choose, for every cube $Q$, a number $c_{Q}$ and estimate the quantity

$$
\sup _{Q} \frac{1}{|Q|} \int_{Q}\left|\log w-c_{Q}\right|
$$

So, let

$$
c_{Q}=\frac{1}{w(Q)} \int_{Q} \log w d w
$$

and

$$
I_{Q}=\frac{1}{|Q|} \int_{Q}\left|\log w-c_{Q}\right|=\frac{w(Q)}{|Q|}\left(\frac{1}{w(Q)} \int_{Q}\left|\log w-c_{Q}\right| w^{-1} d w\right) .
$$

Notice that the $A_{1}^{d w}$ condition for $w^{-1}$ implies that the Hardy-Littlewood maximal function

$$
M_{d w}^{Q} w^{-1}(x)=\sup _{x \in Q^{\prime} \subset Q} \frac{\left|Q^{\prime}\right|}{w\left(Q^{\prime}\right)}
$$

is in $L^{1}(Q ; d w)$ and in fact

$$
\left\|M_{d w}^{Q} w^{-1}\right\|_{L^{1}(Q ; d w)} \leq A_{1}^{d w}\left(w^{-1}\right)|Q|=B_{\infty}(w)|Q| .
$$

So, by a well-known result of Stein [6] (in the context of doubling weights), $w^{-1}$ is in the $L \log L(Q ; d w)$ class, and moreover

$$
\left\|w^{-1}\right\|_{L \log L(Q ; d w)} \leq C D_{w}^{2}\left\|M_{d w}^{Q} w^{-1}\right\|_{L^{1}(Q ; d w)} \leq C D_{w}^{2} B_{\infty}(w)|Q|,
$$

with $C$ independent of $Q$.

On the other hand, since $\log w \in B M O(d w)$, the John-Nirenberg inequality (again, in the doubling context) implies that $\log w-c_{Q}$ is in the $\exp L(Q ; d w / w(Q))$ class and

$$
\left\|\log w-c_{Q}\right\|_{\exp L(Q ; d w / w(Q))} \leq C D_{w}^{2}\|\log w\|_{B M O(d w)},
$$

where $C$ is independent of $Q$.

Applying the generalized Hölder inequality to (2) and then using (37), (4) and (11) we obtain

$$
\begin{aligned}
I_{Q} & \leq C \frac{w(Q)}{|Q|}\left\|\log w-c_{Q}\right\|_{\exp L(Q ; d w / w(Q))}\left(\frac{1}{w(Q)}\left\|w^{-1}\right\|_{L \log L(Q ; d w)}\right) \\
& \leq C D_{w}^{4} B_{\infty}(w)\|\log w\|_{B M O(d w)} \\
& \leq C D_{w}^{4} B_{\infty}(w) \log B_{\infty}(w) .
\end{aligned}
$$

Therefore

$$
\|\log w\|_{B M O} \leq C D_{w}^{4} B_{\infty}(w) \log B_{\infty}(w) .
$$

Now, we have to estimate the doubling constant of $w$ in terms of its $B_{\infty}$ constant. So, let $Q \subset \widetilde{Q}$ be a pair of concentric cubes with $|\widetilde{Q}|=2^{n}|Q|$. Put

$$
\lambda=\frac{2 B_{\infty}(w)}{2 B_{\infty}(w)-1}
$$


and let $\left\{Q_{j}\right\}_{j=0}^{N+1}$ be a finite sequence of cubes such that

$$
Q=Q_{0} \subset Q_{1} \subset \cdots \subset Q_{N} \subset \widetilde{Q} \subset Q_{N+1}
$$

and

$$
\left|Q_{j+1}\right|=\lambda\left|Q_{j}\right|, j=0,1, \ldots, N .
$$

Then

$$
N \sim \frac{n \log 2}{\log \lambda}
$$

By the $B_{\infty}$ condition we have

$$
w(x) \leq B_{\infty}(w) \frac{w\left(Q_{j+1}\right)}{\left|Q_{j+1}\right|}, \text { a.e. on } Q_{j+1} .
$$

Integrating over $Q_{j+1} \backslash Q_{j}$ we obtain

$$
w\left(Q_{j+1} \backslash Q_{j}\right) \leq B_{\infty}(w) w\left(Q_{j+1}\right) \frac{\left|Q_{j+1} \backslash Q_{j}\right|}{\left|Q_{j+1}\right|} .
$$

Equivalently,

$$
w\left(Q_{j}\right) \geq\left(1-B_{\infty}(w)(1-1 / \lambda)\right) w\left(Q_{j+1}\right)=\frac{1}{2} w\left(Q_{j+1}\right) .
$$

Therefore

$$
w(\widetilde{Q}) \leq w\left(Q_{N+1}\right) \leq 2^{N+1} w(Q) \leq C_{n}^{B_{\infty}(w)} w(Q) .
$$

We conclude that

$$
D_{w} \leq C_{n}^{B_{\infty}(w)} .
$$

Since $B_{\infty}(w) \leq \widetilde{C}_{n}$, (15) and (6) imply

$$
\|\log w\|_{B M O} \leq C_{n} \log B_{\infty}(w) .
$$

To finish the proof of the Theorem notice that by the John-Nirenberg inequality there exist $C>0, C_{n}>0$ such that if

$$
0<\lambda<\frac{C_{n}}{\|\log w\|_{B M O}},
$$

then

$$
\frac{1}{|Q|} \int_{Q} e^{\lambda\left|\log w-(\log w)_{Q}\right|} \leq C,
$$

for all cubes $Q$. Note that (7) implies

$$
\frac{C_{n}}{\|\log w\|_{B M O}}>1,
$$

provided that $\widetilde{C}_{n}$ has been chosen close enough to 1 .

Now, for any $p>1+C_{n} \log B_{\infty}(w)$, choose $\lambda$ with

$$
1<\lambda<\frac{C_{n}}{\|\log w\|_{B M O}} \text { and } 1+\frac{1}{\lambda}<p .
$$

Then (8) implies

$$
e^{ \pm \lambda(\log w)_{Q}} \frac{1}{|Q|} \int_{Q} e^{\mp \lambda \log w} \leq C .
$$


Multiplying the \pm estimates we get

$$
\left(\frac{1}{|Q|} \int_{Q} w^{\lambda}\right)\left(\frac{1}{|Q|} \int_{Q} w^{-\lambda}\right) \leq C^{2},
$$

and by Hölder's inequality

$$
\left(\frac{1}{|Q|} \int_{Q} w\right)^{\lambda}\left(\frac{1}{|Q|} \int_{Q} w^{-\lambda}\right) \leq C^{2}
$$

which means

$$
\left(\frac{1}{|Q|} \int_{Q} w\right)\left(\frac{1}{|Q|} \int_{Q} w^{-\lambda}\right)^{\frac{1}{\lambda}} \leq C^{\frac{2}{\lambda}}
$$

This shows that $w \in A_{1+\frac{1}{\lambda}}$, and hence $w \in A_{p}$.

\section{NOTE ADDED IN PROOF}

After acceptance of the paper, the author was informed that the result of the paper was obtained, by similar methods, in the unpublished Ph.D. thesis of A. Politis (University of Chicago, 1995).

\section{REFERENCES}

[1] K. F. Andersen, W. S. Young. On the reverse weak type inequality for the Hardy maximal function and the weighted classes $L(\log L)^{k}$. Pacific J. Math. 112 (1984), no. 2, 257-264. MR.0743983 (85k:42044)

[2] D. Cruz-Uribe, C.J. Neugebauer. The structure of the reverse Hölder classes. Trans. Amer. Math. Soc. 347 (1995), no. 8, 2941-2960. MR1308005 (95m:42026)

[3] B. Franchi. Weighted Sobolev-Poincaré inequalities and pointwise estimates for a class of degenerate elliptic equations. Trans. Amer. Math. Soc. 327 (1991), no. 1, 125-158. MR 1040042 (91m:35095)

[4] J. García-Cuerva, J. L. Rubio de Francia. Weighted norm inequalities and related topics. North-Holland Math. Stud., vol. 116, North-Holland, Amsterdam, 1985. MR0807149 (87d:42023)

[5] B. Muckenhoupt. Weighted reverse weak type inequalities for the Hardy-Littlewood maximal function. Pacific J. Math. 117 (1985), no. 2, 371-377. MR0779926 (86j:42025)

[6] E. M. Stein. Note on the class L $\log L$. Studia Math. 32 (1969), 305-310. MR0247534 (40:799)

Department of Mathematics, University of Crete, Knossos Ave., 71409 Iraklio, Greece

E-mail address: mitsis@fourier.math.uoc.gr 